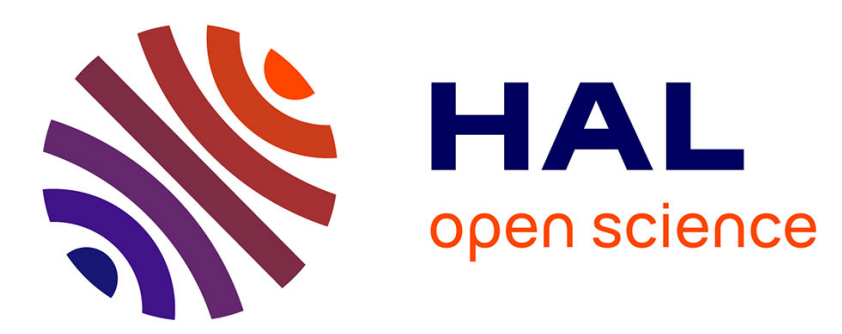

\title{
The plasma-wall transition with collisions and an oblique magnetic field: reversal of potential drops at grazing incidences
}

\author{
J. Moritz, Maxime Lesur, E. Faudot, S. Devaux, S. Heuraux, J. Ledig
}

\section{- To cite this version:}

J. Moritz, Maxime Lesur, E. Faudot, S. Devaux, S. Heuraux, et al.. The plasma-wall transition with collisions and an oblique magnetic field: reversal of potential drops at grazing incidences. Physics of Plasmas, 2019, 26 (1), pp.013507. 10.1063/1.5061832 . hal-02171905

\section{HAL Id: hal-02171905 \\ https://hal.science/hal-02171905}

Submitted on 3 Jul 2019

HAL is a multi-disciplinary open access archive for the deposit and dissemination of scientific research documents, whether they are published or not. The documents may come from teaching and research institutions in France or abroad, or from public or private research centers.
L'archive ouverte pluridisciplinaire HAL, est destinée au dépôt et à la diffusion de documents scientifiques de niveau recherche, publiés ou non, émanant des établissements d'enseignement et de recherche français ou étrangers, des laboratoires publics ou privés. 
The plasma-wall transition with collisions and an oblique magnetic field: reversal of potential drops at grazing incidences.

\author{
J. Moritz, ${ }^{1, a)}$ M. Lesur, E. Faudot, S. Devaux, S. Heuraux, and J. Ledig \\ Institut Jean Lamour, UMR 7198 CNRS - Université de Lorraine, Campus Artem, \\ 2 allée André Guinier, 54011 Nancy, France
}

(Dated: 24 September 2018)

The plasma-wall transition is studied by using 1d3V particle-in-cell (PIC) simulations in the case of a one dimensional plasma bounded by two absorbing walls separated by 200 Debye lengths $\left(\lambda_{d}\right)$. A constant and oblique magnetic field is applied to the system, with an amplitude such that $r<\lambda_{d}<R$, where $r$ and $R$ are the electron and ion Larmor radius respectively. Collisions with neutrals are taken into account and modelled by an energy conservative operator, which randomly reorients ion and electron velocities. The plasma-wall transition (PWT) is shown to depend on both the angle of incidence of the magnetic field with respect to the wall $\theta$, and on the ion mean-free-path to Larmor radius ratio, $\lambda_{c i} / R$. In the very low collisionality regime $\left(\lambda_{c i} \gg R\right)$ and for a large angle of incidence, the PWT consists in the classical trilayer structure (Debye sheath / Chodura sheath / Pre-sheath) from the wall towards the center of the plasma. The drops of potential within the different regions are well consistent with already published models. However, when $\sin \theta \leq R / \lambda_{c i}$ or with the ordering $\lambda_{c i}<R$, collisions can not be neglected, leading to the disappearance of the Chodura sheath. In these case, a collisional model yields analytic expressions for the potential drop in the quasi-neutral region, and explains, in qualitative and quantitative agreement with the simulation results, its reversal below a critical angle derived in the paper, a regime possibly met in the SOL of tokamaks. It is further shown that the potential drop in the Debye sheath slightly varies with the collisionality for $\lambda_{c i} \gg R$. However, it tends to decrease with $\lambda_{c i}$ in the high collisionality regime, until the Debye sheath finally vanishes.

PACS numbers: 52.40.Kh, 52.65.Rr, 52.40.Fd

Keywords: Plasma sheath, PIC simulations, Magnetic confinement

a) jerome.moritz@univ-lorraine.fr 


\section{INTRODUCTION}

Sheaths are space-charged regions that take place at plasma boundaries in order to balance ion and electron losses. The material surface in contact with the plasma can be an electrode, or the wall of any reactor. It becomes negatively charged due to the high velocity of electrons with respect to their positive counterpart. An electric field is built up at the vicinity of the negatively charged wall then repels electrons and attracts ions, giving rise to the non-neutral regions called 'sheaths'.

Sheath formation is of paramount importance for many applications in plasma physics, such as Langmuir probe measurements in low temperature plasma, fabrication processes of nano-materials, objects or thin films ${ }^{1}$, reactors in fusion plasma, where the plasma-wall transition (PWT) can lead to prejudicial heating and erosion of the surface coating ${ }^{2}$, and spacecrafts, where onboard instruments can be affected by surface charging ${ }^{3}$. Sheaths have then been studied theoretically for several decades for the purpose of a better understanding and technological uses.

In the absence of magnetic field, the plasma/wall transition is split into two main regions i.e the non-neutral sheath and the quasi-neutral collisional pre-sheath. The sheath region is known to scale with the Debye length $\lambda_{d}$, while the relevant characteristic length for the pre-sheath can be for instance the collision mean-free-path with neutrals $\lambda_{c}$, or the minimum of the various collision mean-free-paths (ionization, recombination, charge-exchange, etc.) which are relevant to describe the physics of the pre-sheath region.

It has been shown based on a fluid model that, in order to prevent an oscillatory potential distribution at the sheath edge, ions velocity perpendicular to the wall $V_{i x}$ must verify what is known as the Bohm criterion ${ }^{4}$ :

$$
V_{i x}>C_{s}=\sqrt{\frac{T_{e}+T_{i}}{M}}
$$

where $C_{s}$ is the ion sound velocity, $T_{e}$ and $T_{i}$ the electron and ion temperature respectively and $M$ the ion mass ${ }^{5,6}$. Note that in this paper, temperatures will be expressed in energy units only. This sound velocity is also the critical velocity at which quasi-neutrality breaks down in the pre-sheath region ${ }^{7}$ (the plasma approximation stands as long as $V_{i x}<C_{s}$ ), so that it is usually assumed that the Debye sheath entrance is located at the sonic point $S$, where $V_{i x}=C_{s}$. Neglecting inertia for electrons ( $m=0$, where $m$ is the electron mass) and 
ionization within the sheath region, assuming equal ion and electron losses at the wall and using Eq. (1), it is possible to calculate the potential drop between the wall and the sheath edge as:

$$
\Delta \phi_{d}=\frac{T_{e}}{2 e} \ln \left[2 \pi \frac{m}{M}\left(1+\frac{T_{i}}{T_{e}}\right)\right],
$$

which is usually a negative quantity.

In the presence of a magnetic field tilted by $\theta$ with respect to the wall, another sonic point can be derived from the fluid equations. First evidenced by Chodura ${ }^{8}$, an additional quasineutral region appears between the Debye sheath and the collisional pre-sheath, where ions are accelerated from $V_{i x}=C_{s} \sin \theta$ to $C_{s}$, ie. from a point $C$ where the projection of the ion velocity along the field line, $V_{i \|}=C_{s}$, to the point $S$ where the component perpendicularly to the wall, $V_{i x}=C_{s}$.

This region, usually called 'Chodura sheath' or 'magnetic pre-sheath' scales with the ion Larmor radius $R^{9}$. However, when the plasma is collisional enough, if the ion mean-free-path $\lambda_{c i}$ is smaller than $R$, despite the preferential direction of the magnetic field, the plasma flow is isotropized by frequent collisions during the ion cyclotronic period. Some authors have shown that in such a high collisional case, the Chodura sheath disappears and overlaps with the collisional pre-sheath, leading to a classical double layer structure for the $\mathrm{PWT}^{10,11}$. Other studies emphasized the role of the magnetic field angle and strength on the different regions since the original work of Chodura ${ }^{12,13}$.

The potential drop in the Chodura sheath $\Delta \phi_{c h o}$, between point $C$ and point $S$, can be easily calculated by neglecting (like previously for the Debye sheath) particle source and electron inertia, as:

$$
\Delta \phi_{c h o}=\frac{T_{e}}{e} \ln (\sin \theta),
$$

which is also a negative quantity.

Interestingly, as pointed out by Stangeby ${ }^{14}$, the potential drop between the Chodura sheath entrance (point $C$ ) and the wall, which we call $\Delta \phi_{T}$, is strictly equal to Eq. (2):

$$
\Delta \phi_{T}=\Delta \phi_{c h o}+\Delta \phi_{d}=\frac{T_{e}}{2 e} \ln \left[2 \pi \frac{m}{M}\left(1+\frac{T_{i}}{T_{e}}\right)\right],
$$

which is the total potential drop in the Debye and the Chodura sheaths. It is expected to be independent on the incidence of the magnetic field as long as one can assume strongly 


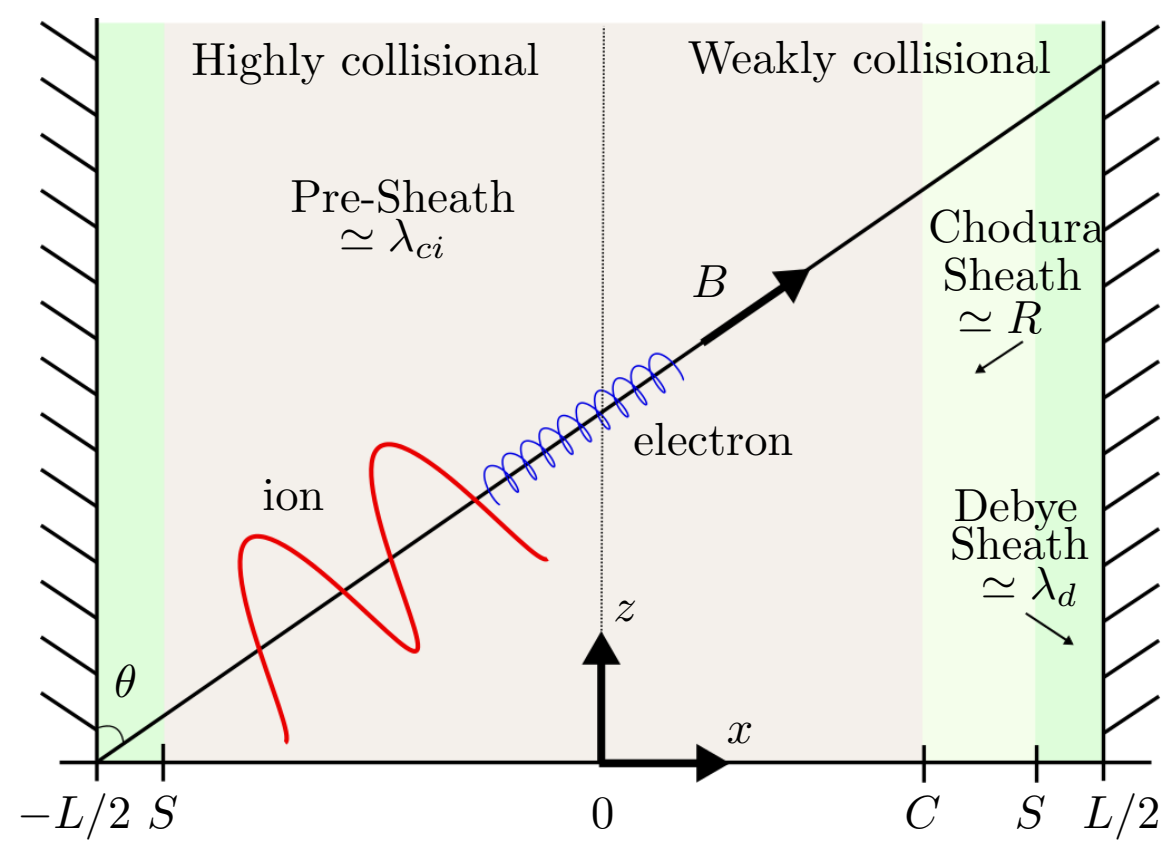

FIG. 1. Sketch of the one dimensional plasma studied, depicting two extreme collisional cases. On the left, the expected layers in the high collisionality regime are the Debye sheath and the collisional pre-sheath. On the righ, in the low collisionality mode, three layers are expected, where the Chodura sheath takes place between de Debye one and the pre-sheath. Each layer scale with its proper characteristic length. The walls are located at the abscissa $-L / 2$ and $L / 2$ and grounded in the PIC simulations. $C$ and $S$ are the sonic points locations. The electron and ion trajectories, drawn in red and blue respectively, are not representative of the real motion of particles, specially in the presence of collisions, where particles undergo a random-walk.

magnetized electrons (barely drifting from their field line) and neglect their inertia. Then for a critical angle $\theta^{*}$, the Debye sheath disappears $\left(\Delta \phi_{d}=0\right)$. It comes from (3) and (4):

$$
\sin \theta^{*}=\sqrt{2 \pi \frac{m}{M}\left(1+\frac{T_{i}}{T_{e}}\right)} .
$$

Below $\theta^{*}$ (of the order of $9.12,6.43$ and $4.75^{\circ}$ for $M / m=500,1000$ and 1836 respectively and assuming $T_{i}=T_{e}$ ), quasi-neutrality does not break down anymore and the Bohm criterion given by Eq. (1) is not fulfilled. Stangeby ${ }^{15}$ inferred that below $\theta^{*}$ the potential drop in the Chodura sheath still equals Eq. (4) as long as $\theta \geq \sqrt{m / M}$, and estimated the ion fluid velocity at the wall (at the Chodura sheath exit) as proportional to $\sin \theta$. This 
tendency has been checked against kinetic simulations, where it was shown that charge separation progressively vanishes for grazing incidence, with the ion flow velocity limited to subsonic speeds ${ }^{16}$. However these kinetic simulations were performed with electrons following a Boltzmann law, so that their inertia was not taken into account, leading to a possible discrepancy at grazing incidences. This regime of very grazing incidence, where $\theta<\theta^{*}$, despite its strong implication in tokamaks for instance, has never been deeply investigated for inertial electrons and in the presence of collisions.

Note that in the limit of $\theta \rightarrow 0$, in a $1 \mathrm{~d} / 3 \mathrm{~V}$ description of the plasma, the particle flux at the wall is expected to cancel in the collisionless limit because particles can not drift perpendicular to the field line. Some authors have addressed this quasi-static issue theoretically and by using particle-in-cell (PIC) simulations ${ }^{17-24}$. They showed that the potential drop at the vicinity of the surface is opposite, ie. ions are pushed back into the plasma instead of being accelerated towards the walls, the space charge being negative, due to the larger Larmor radius of the ions (vs the electrons one). Collisions can restore the particle current perpendicularly to the field line though, for $\theta=0$, and the potential drop sign will then depend on the mean-free-path to Larmor radius ratio ${ }^{25}$.

In this paper we investigate by means of PIC simulations, without assuming Boltzmann electron response, the evolution of the different potential drops in the PWT with respect to both the angle of incidence of the magnetic field and the charged particles vs. neutrals collision rates. In a first part of the paper, after a description of the geometry of the studied system and a general overview of the PIC code, potential and velocity spatial profiles, followed by the potential drops in the PWT, are presented for a large range of the meanfree-path to Larmor radius ratios. In a second part, we review the fluid models allowing the derivation of points $C$ and $S$; we also include the electron inertia in order to extrapolate potential drops at very grazing incidences and derive a modified Bohm criterion. We show that for large $\theta$, the simulated potential drop in the combined collisional pre-sheath and Chodura sheath follows its expected fluid angular variation. We also show that under a critical incidence angle given by $\theta_{c}=\arcsin \frac{R}{\lambda_{c i}}$, when $\lambda_{c i}>R$, particle flows depend on collisions just as in the high collisionality case when $\lambda_{c i}<R$. Potential drops in the quasineutral region, calculated by using a collisional model, reproduce fairly well the simulated ones when $\theta<\theta_{c}$ or $\lambda_{c i}<R$. 


\section{PIC SIMULATIONS}

The studied system is in a one dimensional plasma bounded by two conductive walls, separated by $200 \lambda_{d}$, with the origin of the $x$ axis in the center of the plasma, as depicted in Fig. 1. The magnetic field, of strength $B$, is tilted by $\theta$ with respect to the wall in the $(O z)$ direction. The 1d3V PIC code used for the simulations was developed in the laboratory by the authors ${ }^{24}$. The simulation cell size is chosen as $0.1 \times \min \left(r, \lambda_{d}\right)$, where $r$ is the electron Larmor radius, in order to describe with a sufficient accuracy the motion of both ions and electrons. For all the simulations presented in this study we choose $T_{i}=T_{e}=2 \mathrm{eV}$, $M / m=500$ and $B=0.05 \mathrm{~T}$. With such a magnetic field, we have $r / \lambda_{d}=0.9$, so we can consider that electrons are strongly magnetized and barely drift from their field line.

Initially, the superparticles are uniformly distributed on the grid and their velocity chosen randomly from Maxwellian distributions, whose nominal temperatures are $T_{i}$ and $T_{e}$. During the simulations runs, the number of ions is kept constant by the following method: at each time step, couples (ion + electron) are injected at random positions in the plasma, in order to compensate for the number of ions lost at both walls during the previous time step.

The charged particles undergo collisions with the neutrals. We have developed a simple operator, which conserves the total kinetic energy as well as the total momentum, assuming particles as hard spheres, with a cross-section independent of the velocity. A complete description of the collisional model is given in reference ${ }^{25}$. Note that within this hard sphere model, assuming ions and neutrals of identical diameter, the ion mean-free-path $\lambda_{c i}=\lambda_{c e} / 4$, with $\lambda_{c e}$ the electron one. It is also important to note here that, this collisional model makes faster particles have higher probability to collide (the cross-section being independent of the velocity). Moreover the injection method used, which is known to distort the velocity distribution functions ${ }^{26-28}$, induces a cooling of the plasma with respect to the nominal loaded one. That is why the real temperature of the charged particles to which we normalize potential drops and velocities at the end of the simulations, are extracted from the PIC simulations via a Maxwellian fit of the velocity distribution functions.

The electron mean-free-path $\lambda_{c e}$ is set such as electrons are not demagnetized by collisions, within the range $10<\frac{\lambda_{c e}}{r}<750$. As previously explained, the ion one is 4 times smaller, therefore, using the nominal temperatures and the mass ratio, it satisfies the ordering $0.1<$ $\frac{\lambda_{c i}}{R}<8.3$. This range of the ion mean-free-path allows the study of the transition between 
a highly collisional regime, where the magnetic field effect onto ion motion is canceled by collisions, to an anisotropic one, where the ion flow has to follow the field line. This transition can be seen on the spatial potential and velocity profiles as depicted in Fig. 2.
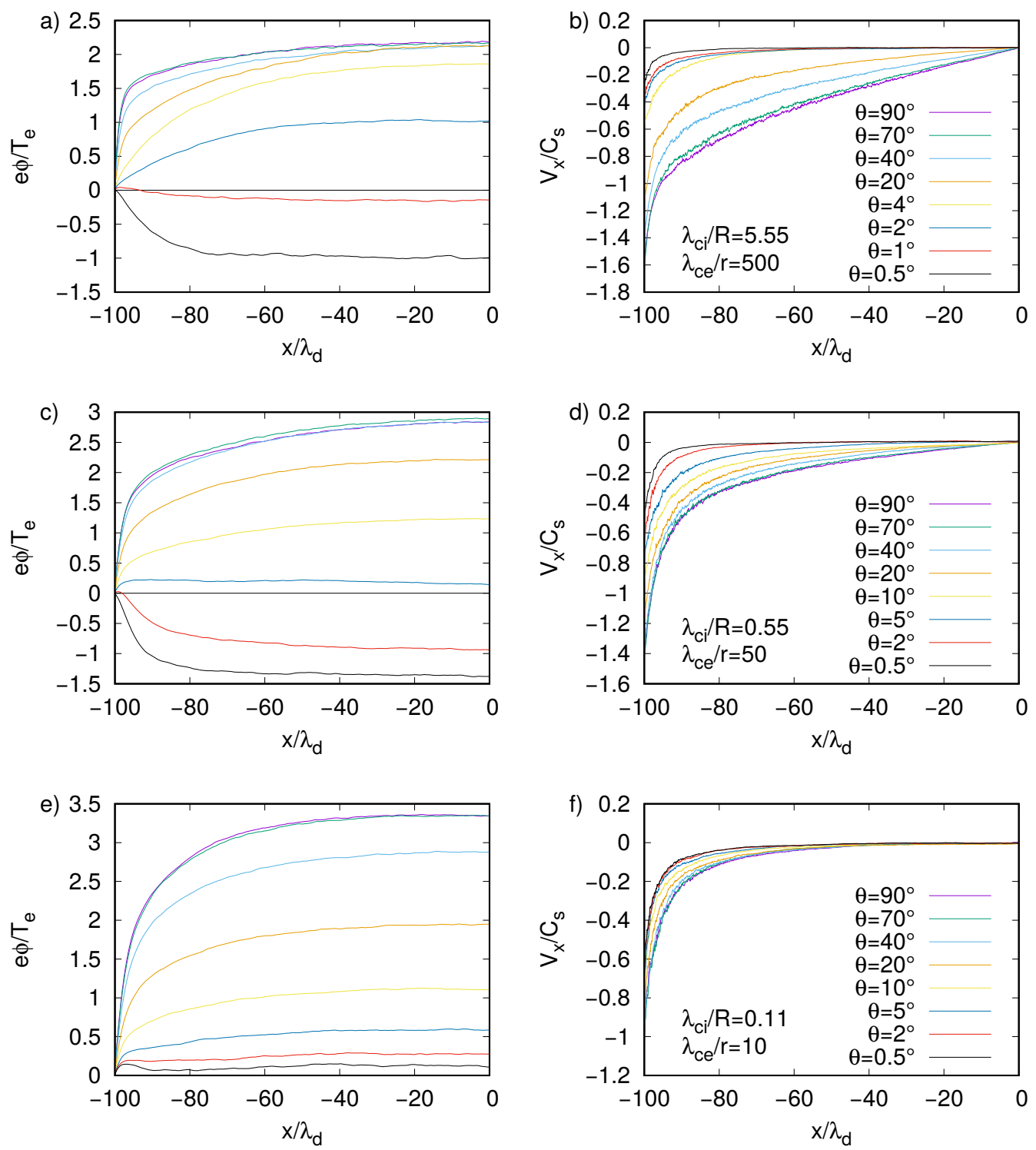

FIG. 2. Left: Normalized potential profiles for 3 different mean-free-path to Larmor radius ratios and 8 values of the angle of incidence of the magnetic field $\theta$. Right: corresponding ion velocity profiles normalized to the sound velocity.

In the very high collisional case $\left(\lambda_{c e} / r=10, \lambda_{c i} / R=0.11\right)$, the magnetic field incidence does not change the ion velocity flow qualitatively (see Fig. 2f): the subsonic flow is isotropic for ions and the potential in the plasma is always positive, so ions are accelerated towards 
the collecting surface for any $\theta$ as depicted in Fig. 2e. For intermediate neutral gas density, $\lambda_{c e} / r=50$ and $\lambda_{c i} / R=0.55$, the effect of the magnetic field begins to influence ion flow as seen in Fig. 2d. In that case, for grazing incidences of the magnetic field, when $\theta<5^{\circ}$, the opposite situation than previously arises, since it is necessary to push back ions into the plasma (the potential drop between the wall and the plasma is inverted in Fig. 2c). This situation has already been evidenced in the case of a magnetic field parallel to the wall ${ }^{25}$. For larger incidences though, the potential drop between the wall and the center of the plasma column reaches $-2.9 T_{e} / e$ for $\theta=90^{\circ}$ and the ion flow is supersonic for $\theta \geq 20^{\circ}$. The inversion of the potential drop between the wall and the center of the plasma can also be seen on the space charge profiles in Fig. 3a for $\lambda_{c i} / R=0.55$. The space charge amplitude decreases with $\theta$ as already pointed out in reference ${ }^{16}$, and for $\theta=0.5^{\circ}$, the space charge is alternatively positive close to the wall and negative towards the plasma, as in the case of a perfectly aligned magnetic field ${ }^{25}$. It also leads to an inversion of the electric field $E$ polarity as shown in Fig. 3b, where ions are accelerated towards the wall for $\theta \geq 5^{\circ}$, and pushed back into the plasma for very grazing incidences otherwise.

Finally, when both ions and electrons are magnetized (ie. $\lambda_{c e} / r=500$ and $\lambda_{c i} / R=5.5$ ), there is a strong dependence of the ion velocity flow on the magnetic field incidence (Fig. $2 \mathrm{~b})$. It stays supersonic at the vicinity of the wall for $\theta>20^{\circ}$ and the velocity at the exit of the plasma decreases slowly with $\theta$ (see also Fig. 7b). The same observation on the potential profiles in Fig. 2a can be done as previously, although the potential drop between the wall and the plasma center reaches $-2.17 T_{e} / e$ for large incidences. This is slightly smaller than for $\lambda_{c i} / R=0.55$ in Fig. 2c, which, we infer, is due to the lower collisionality (it is more difficult to increase the velocity flow, when the friction is more important, so a larger potential drop is required).

In order to define the potential drops in the different parts of the PWT, we use a simple criterion that can be applied to all PIC results: we consider that the Debye sheath entrance is located at the sonic point $S$, where $V_{i x}=C_{s}$. If the ion velocity flow does not reach the sonic point, we assume that the Debye sheath disappears and $\Delta \phi_{d}=0$. Otherwise, the spatial coordinate of $S$ is obtained from $V_{i x}$, for instance from Fig. 2b, and the corresponding potential value $\phi(s)$, from the normalized potential profile (eg. Fig. 2a).

Fig. 4 shows the potential drop within the Debye sheath against $\theta$ for different meanfree-path to Larmor radius ratios. In the very high collisionality case, for $\lambda_{c e} / r=10$ ie. 

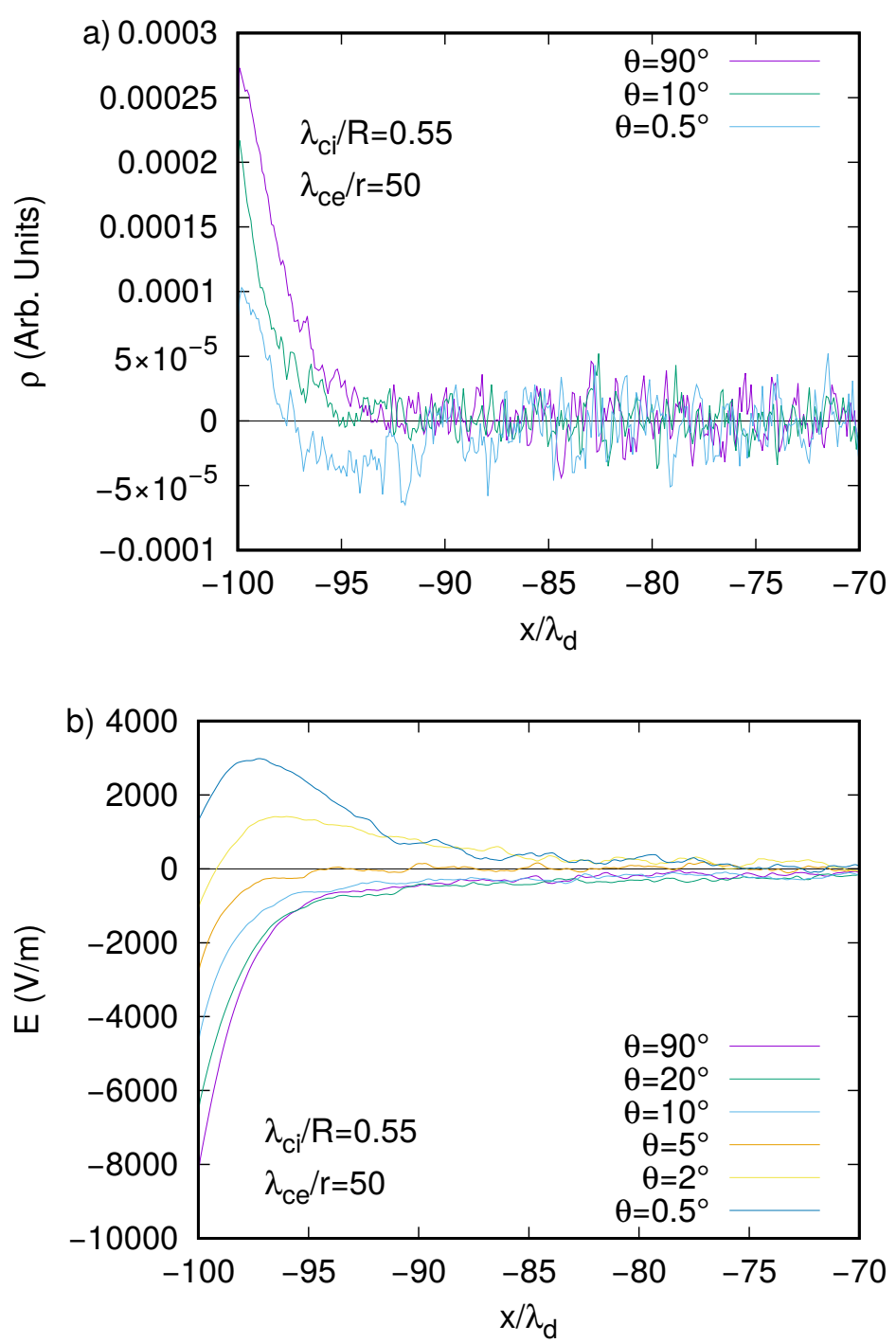

FIG. 3. a) Space charge at the vicinity of the left wall for 3 angles of incidence and a medium collisionality of the ions $\left(\lambda_{c i}=0.55\right)$. b) Electric field profiles for the same mean-free-path to Larmor radius ratio as in a) for 6 angles of incidence of the magnetic field.

$\lambda_{c i} / R=0.11$, the ion velocity flow does not reach $C_{s}$, and there is no Debye sheath (Fig. 4a). In this case, the whole potential drop between the wall and the center of the plasma is in the collisional pre-sheath, which is quasi-neutral. For larger mean-free-paths in Fig. 4a and in Fig. 4b, $\Delta \phi_{d}$ increases with $\lambda_{c e} / r$ until its saturation for $\lambda_{c e} / r>88\left(\lambda_{c i} / R>0.98\right)$ in the medium-low collisionality regime of the ions. In this case, a high space-charge electric field is required to balance ions and electrons losses at the walls.

Using Eq. (4) with the nominal plasma parameters, it comes that for $\theta \rightarrow \pi / 2$, 

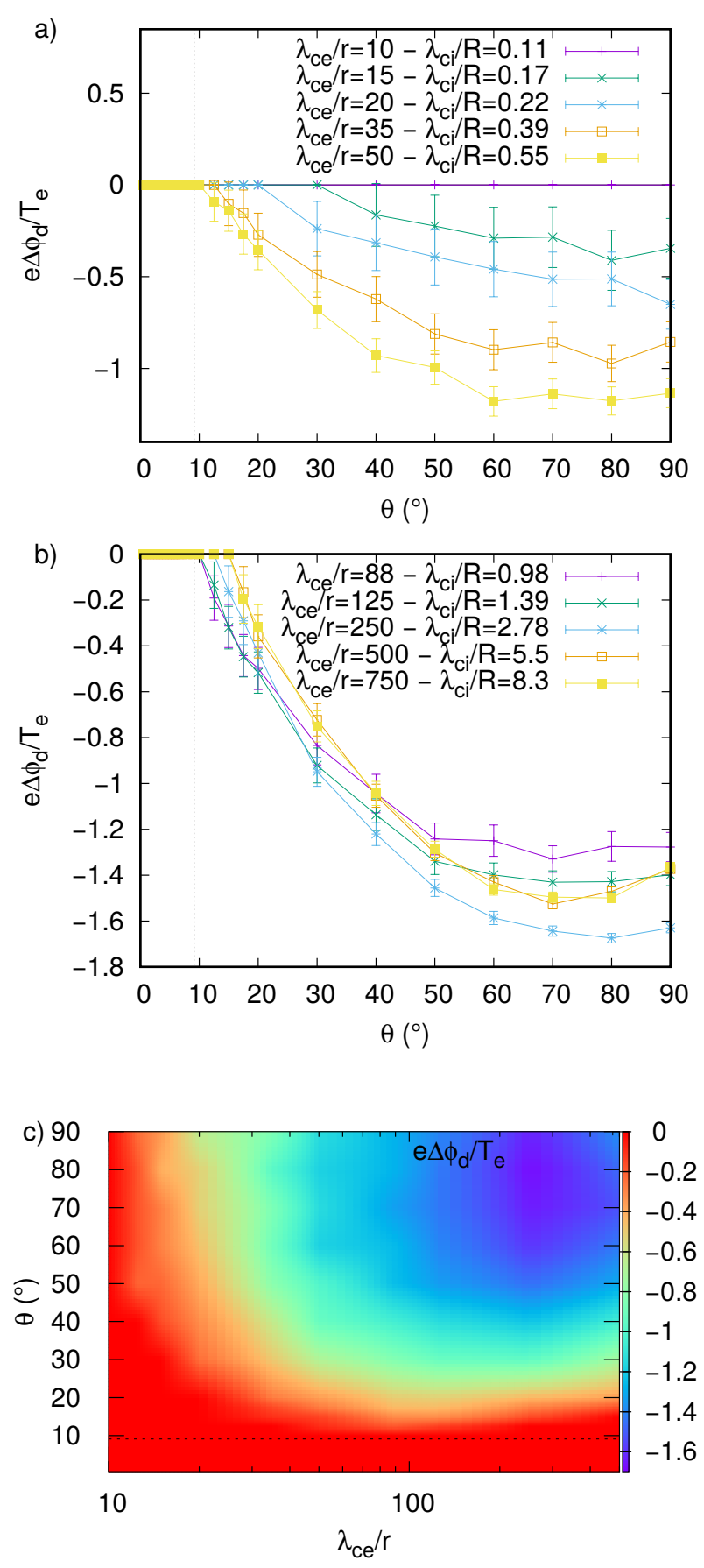

FIG. 4. Normalized potential drop $\Delta \phi_{d} / T_{e}$ in the Debye sheath against the incidence of magnetic field $\theta$ for different mean-free-path to Larmor radius ratios. In a), the high collisionality makes the ion velocity flow isotropic, while in b) both ions and electrons are magnetized. The dotted line is at the abscissa $\theta=\theta^{*}$ given by Eq (5). c) Density plot of $\Delta \phi_{d} / T_{e}$ vs. $\theta$ and $\lambda_{c e} / r$.

$e \Delta \phi_{d} / T_{e} \rightarrow-1.84$, which is comparable to the simulation results in Fig. $4 \mathrm{~b}, e \Delta \phi_{d} / T_{e} \simeq-1.4$ 
for the lowest collisionality (as mentioned previously, the plasma temperature is usually colder than its nominal value at the end of the simulation runs due to the particle injection procedure: this can explain the small discrepancy between the expected value and the simulations one; formula (4) should be used with the temperatures of each simulation run). The dotted line in Fig. 4 represents the critical angle $\theta^{*}$, given by Eq. (5), where the Debye sheath is expected to disappear based on the fluid model. $\theta^{*}$ is always a bit smaller (in the range $3-5^{\circ}$ ) than the angle at which $\Delta \phi_{d}$ really vanishes, due to collisions. This can be seen also in Fig. 4c, which is a density plot representing the potential drop in the Debye sheath vs. $\theta$ and $\lambda_{c e} / r$. The red color indicates the region where the Debye sheath vanishes. We observe that both grazing incidence and high collisionality are responsible of its disappearance. This density plot was obtained by an interpolation of our numerical results, which in large part are shown in Fig. 4a and 4b.

Note that the error bars in Fig. 4, as well as in the following figures, are calculated based on two sources of error. The potential profiles are obtained by averaging the signal in time over several ion cyclotronic periods (just as the velocity profiles or the densities), so it is possible to calculate the standard error of the mean, which is the first source of error. The second one is simply based on the extraction procedure explained previously, when searching for the Debye sheath entrance. This location can not be known at a better precision than the grid step size, which is, with our magnetic field strength, $0.1 \times r$. This gives finally an uncertainty on the potential values.

Once the sonic point $S$ is determined, and its potential value $\phi(S)$ read, one can extract the potential drop in the quasi-neutral region, $\Delta \phi_{q n}$ as $\phi(S)-\phi(0)$, where $\phi(0)$ is the potential at the center of the plasma. If the plasma does not reach the Bohm velocity, $\phi(S)=\phi(-L / 2)=0$ and the potential drop in the quasi-neutral region consists of the total potential variation between the grounded wall and the center of the plasma (which happens for high collisionality or grazing incidence such as $\left.\theta<\theta^{*}\right)$.

The quasi-neutral region can be a collisional pre-sheath only if $\lambda_{c i}<R$. Otherwise it is composed of the Chodura sheath and a collisional pre-sheath as depicted in Fig. 1. Fig. 5a and Fig. 6a show the potential drop $e \Delta \phi_{q n} / T_{e}$ with respect to the angle $\theta$ in the two extreme cases of low and high collisionality respectively. When $\lambda_{c e} / r>250$ (ie. $\lambda_{c i} / R>2.77$ ), in Fig. $5 \mathrm{a}, \Delta \phi_{q n}$ does not vary anymore with the mean-free-path for $\theta>20^{\circ}$. For comparison we plot in the same figure the collisionless case which does not deviate from the low collisional 

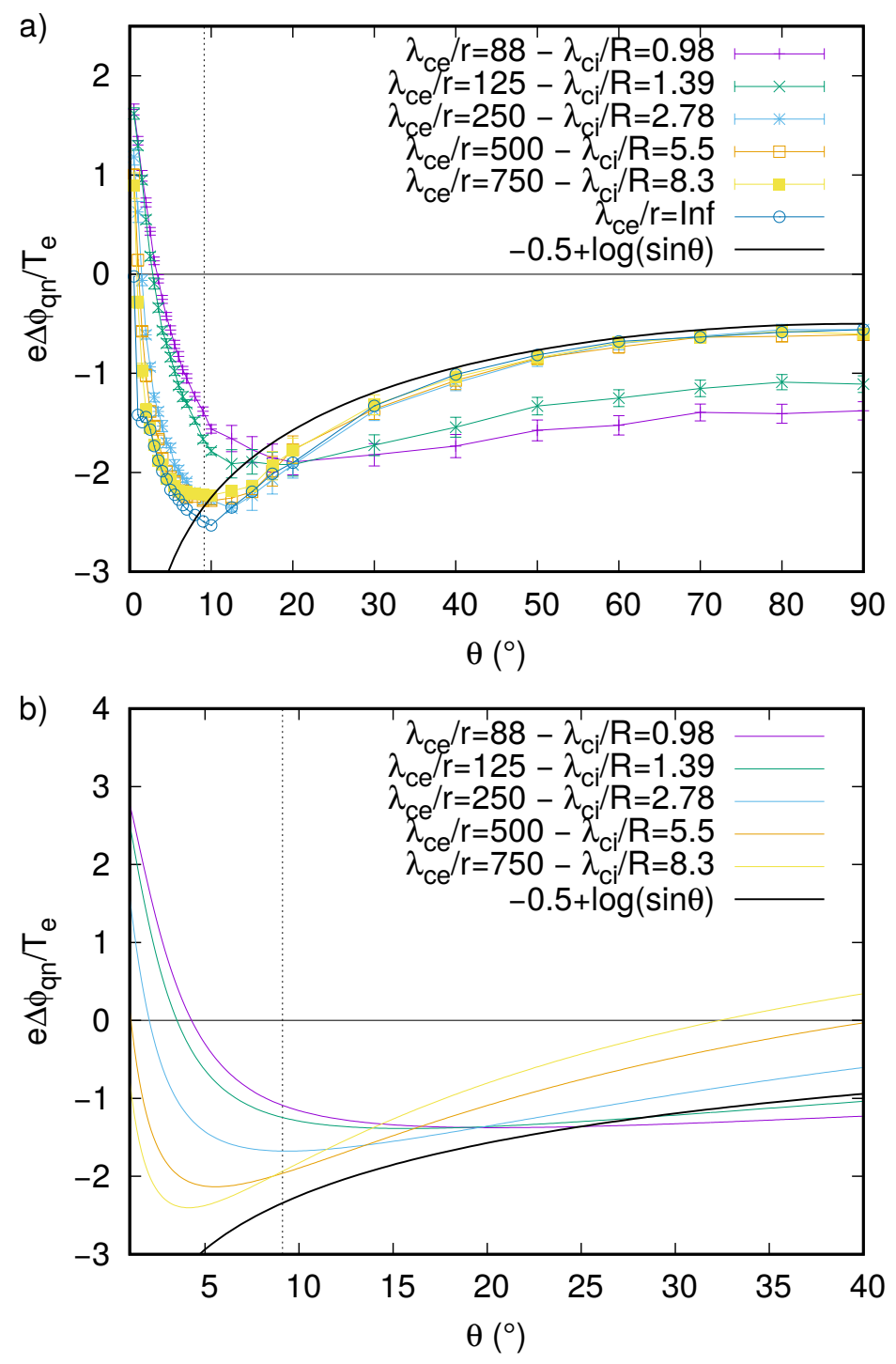

FIG. 5. a) Normalized potential drop $e \Delta \phi_{q n} / T_{e}$ in the quasi-neutral region vs. the angle of incidence of the magnetic field in the medium-low collisionality regime of the ions. b) Theoretical $e \Delta \phi_{q n} / T_{e}$, Eq. (33), in the context of a high collisionality approximation.

ones $\left(\lambda_{c e} / r=250,500\right.$ or 750$)$. On the other hand, below $\theta=20^{\circ}$, a slight difference occurs between the various curves; more particularly, it can be seen that the change in the sign of $\Delta \phi_{q n}$ appears at angles which decrease with increasing $\lambda_{c e} / r$. In the high collisional case, the potential drop in the quasi-neutral region (ie. the collisional pre-sheath) is very sensitive to the ratio $\lambda_{c e} / r$. The smaller it is, the larger both collisionality and potential drop are (see Fig. 6a). The sign of $\Delta \phi_{q n}$ also changes at grazing incidences, but for larger angles than in the low collisional case. 
Finally, we have extracted the Chodura point location $C$ from the velocity profiles, as we did for the sonic point $S$, and calculated the total potential drop in the combined Chodura and Debye sheaths $\Delta \phi_{T}=\Delta \phi_{d}+\Delta \phi_{\text {cho }}$, which is expected to stay constant, independent on the angle of incidence of the magnetic field (see Eq. (4)). Fig. 7a shows $\Delta \phi_{T}$ vs. $\theta$ for different mean-free-path to Larmor radius ratios, only in the case $\lambda_{c i} \geq R$. For $\theta>20^{\circ}$, the total potential drop keeps a constant value, close to the expected one. However for grazing incidences, in contrary with Stangeby assumptions ${ }^{15}, \Delta \phi_{T}$ varies with the magnetic field incidence (specifically around and below $\theta^{*}$ ), rapidly decreasing and even becoming positive at very low incidences of the order of a few degrees.

In order to explain all these features, and more particularly the behavior of the potential drops in the PWT for grazing incidences, we expanded the fluid models, inspired by Ahedo's study ${ }^{10}$, taking into account both magnetic field and collisional effects on ion fluid velocity, as well as electron inertia.

\section{FLUID MODEL FOR THE QUASI-NEUTRAL REGION}

\section{A. Velocity field in the presence of $B$ and collisions with neutrals}

The magnetic $\vec{B}$ and electric field $\vec{E}$ components are $B \times(\sin \theta, 0, \cos \theta)$ and $E \times(1,0,0)$ respectively.

In the steady state, the fluid equations of momentum conservation on the $x, y$ and $z$ components, denoting the derivative in $x$ by a prime symbol, are :

$$
\begin{gathered}
n \mu V_{x} V_{x}^{\prime}=-n q \phi^{\prime}+n q V_{y} B \cos \theta-n^{\prime} T-n \mu \nu V_{x} \\
n \mu V_{x} V_{y}^{\prime}=-n q V_{x} B \cos \theta+n q V_{z} B \sin \theta-n \mu \nu V_{y} \\
n \mu V_{x} V_{z}^{\prime}=-n q V_{y} B \sin \theta-n \mu \nu V_{z} .
\end{gathered}
$$

This system describes both ions and electrons, where $\mu, n, \nu$ and $q$ are the mass, density, collision frequency and electric charge of the considered species respectively. This set of equations can be rewritten in a more convenient way, which highlights the different lengths of the system: 


$$
\begin{gathered}
\frac{V_{x} V_{x}^{\prime}}{V_{t}^{2}}=-\frac{q \phi^{\prime}}{T}+\frac{V_{y}}{V_{t}} \frac{\cos \theta}{\lambda_{m}}-\frac{n^{\prime}}{n}-\frac{V_{x}}{V_{t}} \frac{1}{\lambda_{c}} \\
\frac{V_{x} V_{y}^{\prime}}{V_{t}^{2}}=-\frac{V_{x}}{V_{t}} \frac{\cos \theta}{\lambda_{m}}+\frac{V_{z}}{V_{t}} \frac{\sin \theta}{\lambda_{m}}-\frac{V_{y}}{V_{t}} \frac{1}{\lambda_{c}} \\
\frac{V_{x} V_{z}^{\prime}}{V_{t}^{2}}=-\frac{V_{y}}{V_{t}} \frac{\sin \theta}{\lambda_{m}}-\frac{V_{z}}{V_{t}} \frac{1}{\lambda_{c}}
\end{gathered}
$$

where $V_{t}=\sqrt{\frac{T}{\mu}}$ is the thermal velocity, and $\lambda_{m}$ and $\lambda_{c}$ the Larmor radius and mean-freepath of the considered species respectively. Neglecting ionization or recombination, the conservation of particles number yields:

$$
\frac{\partial\left(n V_{x}\right)}{\partial x}=0
$$

Extracting $V_{z}$ of Eq. (11), injecting it in Eq. (10), as well as $V_{y}$ from Eq. (10) and in Eq. (9), and using Eq. (12) leads to:

$$
\frac{V_{x}^{\prime}}{V_{t}}-\frac{V_{x}^{\prime} V_{t}}{V_{x}^{2}}=-\frac{q \phi^{\prime} V_{t}}{T V_{x}}-\frac{\cos \theta}{\frac{\lambda_{m}^{2}}{\lambda_{c}^{2}}+\sin ^{2} \theta}\left(\frac{V_{y}^{\prime}}{V_{t}} \frac{\lambda_{m}}{\lambda_{c}}+\frac{V_{z}^{\prime}}{V_{t}} \sin \theta+\frac{\cos \theta}{\lambda_{c}}\right)-\frac{1}{\lambda_{c}}
$$

In the case of low collisionality $\left(\lambda_{m}<<\lambda_{c}\right)$ and when $\sin \theta>>\frac{\lambda_{m}}{\lambda_{c}}$, Eq. (13) can be simplified as:

$$
V_{x}^{\prime}\left(1-\frac{V_{t}^{2}}{V_{x}^{2}}\right)=-\frac{q \phi^{\prime}}{\mu V_{x}}-\frac{V_{z}^{\prime}}{\tan \theta}-\frac{V_{t}}{\lambda_{c} \sin ^{2} \theta}
$$

Using the same ordering of the characteristic lengths, one can see from Eq. (10) and (11) that, in regions of scale $\lambda_{c}, V_{y}<<V_{z}$ and $V_{x} \simeq V_{z} \tan \theta$. Eq. (14) becomes:

$$
\mu V_{x} V_{x}^{\prime}\left(\frac{1}{\sin ^{2} \theta}-\frac{V_{t}^{2}}{V_{x}^{2}}\right)=-q \phi^{\prime}-\mu \frac{V_{x} V_{t}}{\lambda_{c} \sin ^{2} \theta}
$$

If the previous ordering is valid for both ions and electrons, then the plasma is moving along the magnetic field line only. Equation (15) is the momentum conservation equation along the magnetic line projected onto the $x$ axis.

For an incidence of the magnetic field such as $\sin \theta \simeq \frac{\lambda_{m}}{\lambda_{c}}$, a component perpendicular to the magnetic line appears in the velocity field, and the previous approximations do not stand anymore. It is however possible to consider, when the plasma is quasi-neutral, that 
the plasma velocity is smaller than the thermal one, so that inertial terms in Eq. (9) to (11), quadratic in velocity, can be neglected.

Finally, in the high collisional regime where $\lambda_{c}<\lambda_{m}$, it comes from Eq. (10) and (11) that $V_{y} / V_{x} \simeq V_{z} / V_{x}<<1$. Collisions overcome magnetic order and Eq. (9) describes a diffusive motion along the $x$ axis only.
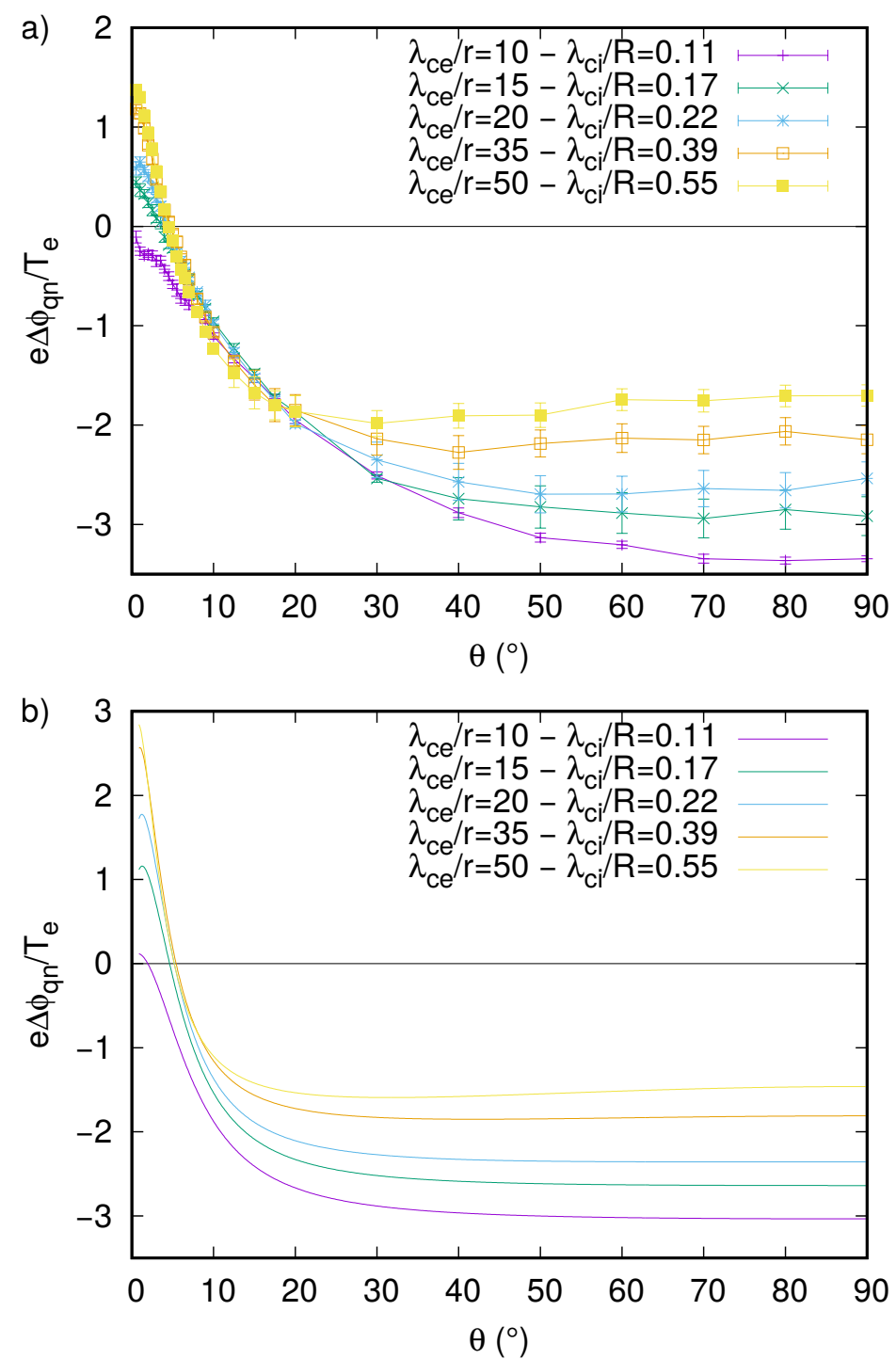

FIG. 6. a)Normalized potential drop $e \Delta \phi_{q n} / T_{e}$ in the quasi-neutral region vs. the angle of incidence of the magnetic field in the high collisionality regime of the ions. b) Theoretical $e \Delta \phi_{q n} / T_{e}$, Eq. (33), for the same mean-free-path to Larmor radius ratios than in a). 


\section{B. Bohm criterion for strongly magnetized electrons}

Let us assume that ion velocity field follows Eq. (9) which stands for any collisionality. Let us further assume $\sin \theta>>\frac{r}{\lambda_{c e}}$ so that Eq. (15) describes electrons momentum conservation for any mean-free-path, but not necessarily ion momentum conservation, because $\sin \theta$ can be larger or smaller than $\frac{R}{\lambda_{c i}}$. In the plasma, far from the sheaths, the plasma approximation stands and we have $n_{i} \simeq n_{e} \simeq n$, where $n_{i}$ and $n_{e}$ are the ion and electron density respectively. It comes that $V_{e x} \simeq V_{i x} \simeq V_{x}$, where $V_{e x}$ is the electron velocity perpendicular to the wall. Injecting the electric force $q \phi^{\prime}$ from Eq. (15) into Eq. (9) and using Eq. (12), with electrons and ions parameters for the mass, temperature, Larmor radius and mean-free-path, yields:

$$
\frac{M \sin ^{2} \theta+m}{T_{i} \sin ^{2} \theta} \frac{V_{x}^{\prime}}{V_{x}}\left(V_{x}^{2}-C_{s \theta}^{2}\right)=\frac{V_{i y} \cos \theta}{V_{i} R}-\frac{V_{x}}{V_{i}}\left(\frac{V_{e} m}{V_{i} M} \frac{1}{\lambda_{c e} \sin ^{2} \theta}+\frac{1}{\lambda_{c i}}\right),
$$

with the modified Bohm velocity:

$$
C_{s \theta}=\frac{C_{s} \sin \theta}{\sqrt{\sin ^{2} \theta+m / M}}
$$

The modified Bohm velocity takes into account electron inertia, that becomes important

for grazing incidences when $\theta \simeq \sqrt{m / M}$ as already mentioned by Stangeby in reference ${ }^{15}$. Then as long as electrons are moving along the field line (for $\lambda_{c e}>>r$ ), even if their inertia matters, the quasi-neutrality breaks down at the modified Bohm velocity, which is very close to $C_{s}$ for large incidences, but vanishes as $\theta \simeq 0$. This result does not depend on the collisionality of the ions, their velocity field could be isotropic for $\lambda_{c i}<R$ or anisotropic when the magnetic effects overcome collisions.

\section{Low collisionality $\lambda_{c i}>>R$}

In regions that scale with $\lambda_{c i}$ and when the angle of incidence of the magnetic field is such as $\sin \theta>\frac{R}{\lambda_{c i}}$, ions also verify Eq. (15), like electrons. Combining both equations and eliminating the electric field force gives:

$$
\frac{m+M}{\sin ^{2} \theta} \frac{V_{x}^{\prime}}{V_{x}}\left(V_{x}^{2}-\frac{T_{e}+T_{i}}{m+M} \sin ^{2} \theta\right)=-\frac{V_{x}}{\sin ^{2} \theta}\left(\frac{m V_{e}}{\lambda_{c e}}+\frac{M V_{i}}{\lambda_{c i}}\right)
$$

In order to get a positive gradient of the velocity, one must have: 


$$
V_{x}<\sqrt{\frac{T_{e}+T_{i}}{m+M}} \sin \theta \simeq C_{s} \sin \theta,
$$

which is known as the Chodura sheath entrance condition. As already pointed out by Ahedo $^{10}$, at this specific sonic point, the plasma enters a steeper region of scale $R$, where it stays quasi-neutral until it enters the Debye sheath at the sonic point $V_{x}=C_{s \theta}$.

\section{High collisionality $\lambda_{c i}<<R$ or incidences such as $\sin \theta<\frac{R}{\lambda_{c i}}$}

When the angle of incidence of the magnetic field is smaller or of the same order of magnitude than $\theta_{c}=\arcsin \frac{R}{\lambda_{c i}}$, it is not possible to neglect the collisional term in Eq. (10) or Eq (11) vs. the magnetic ones. The inertial terms can be disregarded though, for velocities smaller than the thermal one. This situation corresponds geometrically to the interception of the ion Larmor radius with the wall at a distance $\lambda_{c i}$ from it along the field line. In such a case, the Chodura sheath, which extends over some $R$ in front of the wall, becomes collisional, even if $\lambda_{c i}>R$, and tends to disappear and merge with the collisional pre-sheath.

For $\lambda_{c i}<<R$, condition (19) vanishes and so does the Chodura sheath (ions are demagnetized by collisions) and one can see from Eq. (10) and Eq. (11) that, as already mentioned, $V_{i z} \simeq V_{i y}<<V_{i x}$.

In both situations described in this section, Eq. (16) still holds as well as the modified Bohm criterion given by Eq. (17). The important conclusion is that the Chodura sheath merges with the collisional pre-sheath for $\lambda_{c i}<R$ and for incidences such as $\sin \theta<\frac{R}{\lambda_{c i}}$.

\section{E. Potential drop in the quasi-neutral region}

\section{1. $\lambda_{c i}>>R$}

For $\theta$ larger than both $\theta_{c}$ and $\theta^{*}$ and low collisionality, one can neglect electron inertial effects. Indeed dividing successively Eq. (15) for electrons by $m$ and then by $V_{e}^{2}$, using Eq. (12), yields:

$$
\frac{V_{x} V_{x}^{\prime}}{V_{e}^{2} \sin ^{2} \theta}+\frac{n^{\prime}}{n}=\frac{e \phi^{\prime}}{T_{e}}-\frac{V_{x}}{V_{e}} \frac{1}{\sin ^{2} \theta \lambda_{c e}}
$$


Assuming the electron fluid velocity smaller than $V_{e}$, ie. $V_{x} / V_{e}<<1$, and large incidences of the magnetic field, yields the Boltzmann relation from Eq. (20):

$$
\frac{n^{\prime}}{n}=\frac{e \phi^{\prime}}{T_{e}}
$$

Noting the potential at the Debye and Chodura sheath entrance as $\phi_{d}$ and $\phi_{c}$ respectively, it comes from Eq. (21) that $\frac{n_{d}}{n_{c}}=\exp \frac{e\left(\phi_{d}-\phi_{c}\right)}{T_{e}}$, with $n_{d}$ and $n_{c}$ the plasma density at the latter entrances. Knowing the velocity at both sonic points, it is straightforward to calculate the well-known potential drop in the Chodura region using Eq. (12) as:

$$
\Delta \phi_{\text {cho }}=\phi_{d}-\phi_{c}=\frac{T_{e}}{e} \log (\sin \theta)
$$

In order to get the total potential drop in the quasi-neutral region $\Delta \phi_{q n}$, one has to evaluate the potential at the Chodura point, assuming $\phi(0)=0$ and $V_{x}(0)=0$. As explained previously, for such an ordering of the different characteristic lengths, the plasma flows parallel to the field line (ions and electrons). So for every angle, the plasma has to be accelerated from an expected null velocity at $x=0$ to $C_{s}$ at $x=C$ along the field line (or $C_{s} \sin \theta$ in the $x$ direction).

Using Eq. (15) for ions and Eq. (12) and neglecting the collisional drag, we have:

$$
\frac{n^{\prime}}{n}=-\frac{e \phi^{\prime}}{T_{i}}-\frac{M V_{x} V_{x}^{\prime}}{T_{i} \sin ^{2} \theta}
$$

that can be injected in Eq. (21). After integration between the Chodura sheath entrance and the plasma center, it comes that $\phi_{c}-\phi(0)=-0.5 T_{e}$, which is independent of the magnetic field incidence.

The total potential drop in the quasi-neutral region is then, for $\theta>\theta^{*}$ and $\theta>\theta_{c}$ :

$$
\Delta \phi_{q n}=\frac{T_{e}}{e}(\log (\sin \theta)-0.5)
$$

In Fig. 5a, we observe that Eq. (24) fits qualitatively the PIC simulations results for incidences larger than $20^{\circ}$ as long as $\lambda_{c e} / r \geq 250$. Note that the values of $\theta_{c}=\arcsin \frac{R}{\lambda_{c i}}$ for $\lambda_{c e} / r=250,500$ and 750 are 20.96, 10.3 and $6.8^{\circ}$ respectively. Below this threshold value of the incidence of the magnetic field, the collisional model is expected to apply. 

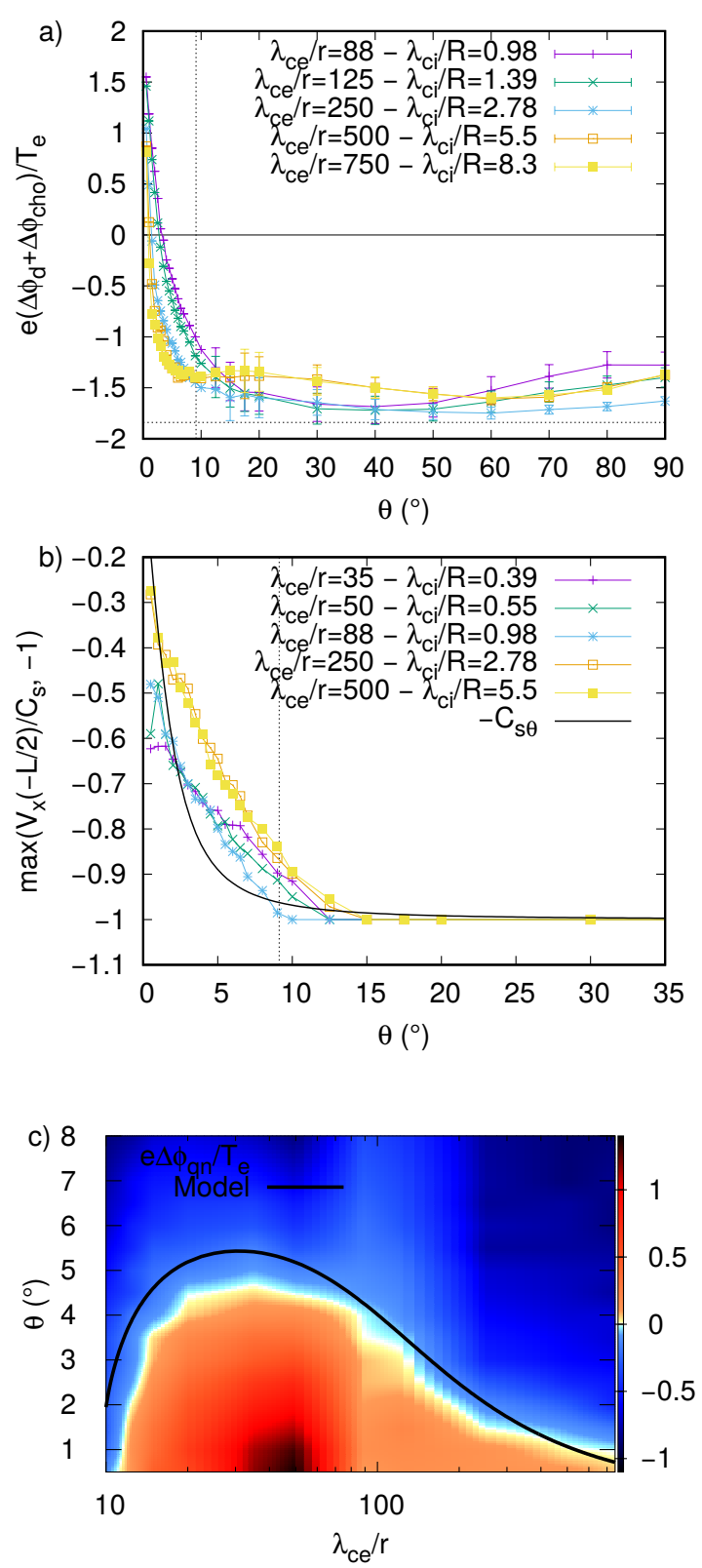

FIG. 7. a) Normalized potential drop $e \Delta \phi_{T} / T_{e}$ in the combined Debye and Chodura sheaths vs. the angle of incidence of the magnetic field in the medium-low collisionality regime of the ions. b) Variation of the velocity at the exit of the quasi-neutral region, that can be the Bohm velocity $C_{s}$ or the velocity at the wall $V_{x}(-L / 2)$, normalized to $C_{s}$. c) Density plot of the potential drop in the quasi-neutral region vs. $\theta$ and the mean-free-path to larmor radius ratio for grazing incidences only. The straight line is the critical angle $\theta_{p}$ plotted using Eq. (35) 


\section{2. $\lambda_{c i}<<R$ or $\sin \theta<\frac{R}{\lambda_{c i}}$}

In such a case, as explained previously, the Chodura sheath disappears, and the entire potential drop in the quasi-neutral region occurs between the sonic point defined by Eq. (17) and the center of the plasma, where it is assumed $V_{x} \simeq 0$. In order to evaluate this

potential drop, we have to neglect the inertial terms in the set of Eqs. (9)-(11) for both ions and electrons and to assume a source term in the plasma. This procedure yields:

$$
V_{i x}=\frac{\mu_{i}}{C_{i}} E-\frac{D_{i}}{C_{i}} \frac{n^{\prime}}{n}
$$

with,

$$
C_{i}=\frac{\nu_{i}^{2}+\omega_{c i}^{2}}{\nu_{i}^{2}+\omega_{c i}^{2} \sin ^{2} \theta},
$$

and $\nu_{i}$ and $\omega_{c i}$ the ion collision and cyclotron frequencies respectively.

If $\theta \rightarrow \pi / 2$, one recovers the classical result without the magnetic field effect with the mobility $\mu_{i}=\frac{|e|}{\nu_{i} M}$ and the diffusion coefficient $D_{i}=\frac{T_{i}}{\nu_{i} M}$. Applying the same reasoning to electrons, with their own coefficients $C_{e}, \mu_{e}$ and $D_{e}$, assuming in the context of the plasma approximation that $V_{i x} \simeq V_{e x}$, it is possible to solve $E$ as:

$$
E=\frac{D_{i} \frac{C_{e}}{C_{i}}-D_{e}}{\mu_{i} \frac{C_{e}}{C_{i}}+\mu_{e}} \frac{n^{\prime}}{n} .
$$

Now the conservation of particle number is:

$$
\frac{\partial\left(n V_{x}\right)}{\partial x}=S
$$

As explained previously, the ions number is kept constant during the simulations: each time one comes across the walls, a (ion, electron) couple is injected randomly in the plasma so that $S=2 \Gamma_{w} / L$ with $\Gamma_{w}$ the particle flux at a single wall.

Replacing Eq. (27) in (25) gives the particle flux $\Gamma(x)=n V_{x}(x)$ :

$$
\Gamma=-\frac{D_{e} \mu_{i}+D_{i} \mu_{e}}{\mu_{i} C_{e}+\mu_{e} C_{i}} n^{\prime}=-D_{a}^{\theta} n^{\prime}
$$

with $D_{a}^{\theta}$ the ambipolar diffusion coefficient which depends on the incidence of the magnetic field line with respect to the wall. Substituting in Eq. (28) yields:

$$
n^{\prime \prime}(x)=-\frac{2 \Gamma_{w}}{L D_{a}^{\theta}}
$$


The previous differential equation can be solved assuming the boundary conditions $n(L / 2) \simeq n(-L / 2) \simeq 0$ as:

$$
n(x)=\frac{\Gamma_{w} L}{4 D_{a}^{\theta}}\left(1-\frac{4 x^{2}}{L^{2}}\right)
$$

In the steady state, we necessarily have $n(0)=n_{0}$, so that $\Gamma_{w}=\frac{4 D_{a}^{\theta} n_{0}}{L}$. The quasi-neutral regions ends at the sonic point $S$ when the Debye sheath exists, otherwise at the walls if the quasi-neutrality does not break down within the plasma. Let us call $V_{\text {out }}$ the velocity at the exit of the quasi-neutral region, such as $V_{\text {out }}=\left|\max \left(-C_{s}, V_{x}(-L / 2)\right)\right|$, assuming $V_{x}<0$ at the vicinity of the left wall as depicted in Fig. 1. Then when the plasma becomes supersonic before reaching the wall, $V_{\text {out }}=C_{s}$ (the velocity at the wall is necessarily $\left|V_{x}(-L / 2)\right|>C_{s}$ ); else, $V_{\text {out }}=\left|V_{x}(-L / 2)\right|$.

If we neglect the source term in the region separating the wall from the sonic point $S$, we can approximate $\Gamma_{w}=n_{\text {out }} V_{\text {out }}$, with $n_{\text {out }}$ the density at the considered point (sonic point $S$ or the wall), which yields:

$$
\frac{n_{\text {out }}}{n_{0}}=\frac{4 D_{a}^{\theta}}{L V_{\text {out }}}
$$

Integrating Eq. (27) between the point $\left(n_{\text {out }}, V_{\text {out }}\right)$ and the center of the plasma gives finally:

$$
\Delta \phi_{q n}=-\frac{D_{i} C_{e}-D_{e} C_{i}}{\mu_{i} C_{e}+\mu_{e} C_{i}} \ln \frac{4 D_{a}^{\theta}}{L\left|V_{\text {out }}\right|}
$$

Fig. 7b shows the angular variation of the normalized velocity at the exit of the quasineutral region as explained above. For incidences larger than $15^{\circ}$, a space-charge field forms and the quasi-neutrality breaks down at the Bohm velocity. For grazing incidences, the exit velocity becomes $V_{x}(-L / 2)$, which considerably decreases with $\theta$ for all mean-free-paths. In the same figure, we plot the modified Bohm velocity $C_{s \theta}$ (Eq. 17), which is in a qualitative agreement with the simulations results. Other authors have derived a similar variation of the velocity as $C_{s \theta}$ at the exit of the plasma from fluid considerations or kinetic simulations ${ }^{15,16}$. In the following, we will then assume that $\left|V_{\text {out }}\right|=C_{s \theta}$ in the collisional model.

Fig. $6 \mathrm{~b}$ shows that in the high collisionality case $\left(\lambda_{c i}<R\right)$, the collisional model (Eq. (33)) is in good agreement with the PIC simulation results, apart from the very grazing incidences, below $2^{\circ}$, where the calculated potential drop is twice the simulated one. In Fig. $5 \mathrm{~b}$, it can be seen that the collisional model is also in good agreement with the simulated result in the lower collisionality case $\left(\lambda_{c i}>R\right)$ for incidences smaller than $20^{\circ}$, ie. smaller 
than $\theta_{c}$. The model also reproduces very well the slope of $\Delta \phi_{q n}$ vs. $\theta$ for the range of meanfree-paths we investigated, and can explain the change of polarity of the potential drop in the quasi-neutral region. However, just like in Fig. 6b, the model gives a larger potential drop than the simulation results below $2^{\circ}$, although the discrepancy is smaller than in Fig. $6 \mathrm{~b}$.

In fact, for such a small incidence, the potential drop $\Delta \phi_{q n}$ is found to be opposite to the usual one, ie. this is a regime where ions are pushed back into the plasma. The ambipolarity is maintained within the plasma, thanks to the ambipolar electric field, as shown in Fig. 3b. However, at the proximity of the wall, over a distance $\simeq r$, a small positive charge arises because electrons are still the fastest species in this non-collisional limited region of the plasma (see Fig. 3a for $\theta=0.5^{\circ}$ ). There is obviously a potential drop associated to this region scaling with $r$, that we do not treat in a fluid model, and that can explain the discrepancy observed for $\theta<2^{\circ}$.

\section{Ambipolar field transition}

In the collisional pre-sheath, which separates the plasma from the Debye (point $S$ ) or the Chodura sheath (point $C$ ), particles are accelerated via an ambipolar field from a null velocity to the corresponding sonic point. Depending on the angle of incidence of the magnetic field, mean-free-path to Larmor radius ratios, the ambipolar field can be either negative and accelerate ions towards the wall, or positive and push back ions into the plasma as seen in Fig. 3b for instance. The transition between both regimes can be evaluated by using Eq. (27). The sign of the ambipolar field changes when:

$$
D_{i} C_{e}=D_{e} C_{i}
$$

We assume for the sake of generality that $\lambda_{c i}=\gamma \lambda_{c e}$. For a given electron mean-free-path to Larmor radius ratio $\lambda_{c e} / r=\alpha$, we can derive from Eq. (34) a critical angle $\theta_{p}$ under which the ambipolar field is positive as:

$$
\sin ^{2} \theta_{p}=\frac{1-A+\alpha^{2}\left(\beta^{2}-A\right)}{\alpha^{2}\left(A \beta^{2}-1+\alpha^{2} \beta^{2}(A-1)\right)},
$$

where $\beta^{2}=\gamma^{2} \frac{T_{e} m}{T_{i} M}$ and $A^{2}=\gamma^{2} \frac{T_{i} m}{T_{e} M}$. Note that this expression stands for any mean-freepath, for any collisional regime of both ions and electrons. Indeed, it is possible to assume 
that in the center of the plasma, the velocity gradient is very small and that all left members of the set of equations (9) to (11) can be neglected for each species.

Fig. 7c is a density plot, interpolated from our simulations results as explained previously, which depicts the normalized potential drop in the quasi-neutral region with respect to $\theta$ and the ratio $\lambda_{c e} / r$, the white contrast in the figure being associated with a null potential drop. For such a null $\Delta \phi_{q n}$, the ambipolar field is expected to change its direction. The critical angle $\theta_{p}=f\left(\lambda_{c e} / r\right)$ from Eq. (35) is also plotted in the figure and it appears that it follows fairly well the white contrast of the density plot. This shows that the collisional model explains the transition in the ambipolarity, which is seen in Fig. 3b. Note that this unexpected regime, where ions have to be slowed down with respect to electrons, appears at very grazing incidences or high collisionality; it does not coexist with a Debye sheath as seen in the density plot of Fig. 4c.

\section{CONCLUSION}

In this paper, we studied by mean of PIC simulations the evolution of the potential drops in the different layers constituting the PWT, in the presence of a magnetic field tilted by $\theta$ with respect to the wall, and of collisions with neutrals. We investigated a large range of collisionality for the ions, from $\lambda_{c i} / R=0.11$ to 8.3 , which was large enough to study the transition between two opposite regimes: one where the magnetic order was destroyed by collisions, to another one, where both ions and electrons were moving along the field line in the plasma. We showed that both the collisionality and the incidence of the magnetic field have important influences on the PWT characteristics, from the nonneutral Debye sheath to the quasi-neutral region. We evidenced that in the high collisional regime, the Debye sheath disappears, the plasma being subsonic for any incidence, because the potential drop in the collisional pre-sheath is large enough to balance ions and electrons losses at the walls. When the ion mean-free-path increases and collisions with neutral become less and less frequent, the potential drop in the Debye sheath increases, because a space-charge field is needed to accelerate ions and slow down electrons. However when the incidence decreases, ion mobility towards the wall increases with respect to electrons, due to collisions. That is why the potential drop in the Debye sheath decreases with $\theta$, which is expected in the collisionless limit because of the particle flux reduction at the wall; here 
the effect is exacerbated by collisions. Concerning the quasi-neutral region, we evidence two trends: when the ion mean-free-path is very large with respect to the ion Larmor radius $\left(\lambda_{c i}>>R\right)$, the plasma flows parallel to the magnetic field line in the pre-sheath, which scales with $\lambda_{c i}$, until it enters the Chodura region. At the exit of the Chodura sheath, the quasi-neutrality breaks down, and the total potential drop between the Debye sheath entrance and the center of the plasma follows the variation $e \Delta \phi_{q n} / T e=\ln (\sin \theta)-0.5$. When the angle of incidence of the magnetic field is such as $\theta \leq \theta_{c}=\arcsin R / \lambda_{c i}$, and although the ion mean-free-path is quite larger than the Larmor radius, the Chodura sheath disappears and merges with the collisional pre-sheath. For such incidences, the potential drop in the quasi-neutral region follows a collisional law, where inertia of both ions and electrons is neglected. The same collisional law successfully models $\Delta \phi_{q n}$ when $\lambda_{c i}<R$, in the regime of high collisionality of the ions. In a plasma reactor such as $\mathrm{ALINE}^{29}$, with an hydrogen plasma such as $T_{e}=T_{i}=2 \mathrm{eV}$ and $B=0.1 \mathrm{~T}$, and an expected elastic collisional frequency for electrons with neutrals of the order of $\nu_{e}=45 \mathrm{MHz}$, we have $\lambda_{c e} / r=276$ or $\lambda_{c i} / R=1.6$, assuming $\lambda_{c e}=4 \times \lambda_{c i}$. In the conditions met in the scrape-off layers (SOL) of tokamaks ${ }^{14,30}$, with $T_{e}=20 \mathrm{eV}$ and $B=2 \mathrm{~T}$, we have $\lambda_{c e} / r=5525$ or $\lambda_{c i} / R=32$ for hydrogen. The critical angle $\theta_{c}$ below which collisions make the Chodura sheath disappear and merge with the collisional pre-sheath is of the order of $38^{\circ}$ for ALINE and $1.78^{\circ}$ for the SOL of tokamaks. This is respectively quite larger and of the same order of magnitude than $\theta^{*}=4.74^{\circ}$, the theoretical angle at which the Debye sheath is expected to vanish in the collisionless limit. Moreover, the critical angle $\theta_{p}$ below which the potential drop in the pre-sheath is expected to reverse, and push-back ions into the plasma, is of $\theta_{p}=2.31^{\circ}$ for a reactor such as ALINE and of $0.136^{\circ}$ for the SOL of tokamaks. Collisions with neutrals, and other phenomenons inducing a similar drift of the particles perpendicularly to the field line (turbulence, anomalous transport, electron-ion collisions, shear velocity...) may then affect significantly the potential drops in the PWT, in both the Debye sheath and the quasi-neutral region, for relative large angles, in plasma reactors with the characteristics of ALINE. The effect would be more subtle for warmer and strongly magnetized plasmas. 


\section{REFERENCES}

${ }^{1}$ P. Chabert and N. Braithwaite, Physics of radio-frequency plasmas (Cambridge University Press, 2011) pp. 1-17

${ }^{2}$ R. A. Pitts, J. P. Coad, D. P. Coster, G. Federici, W. Fundamenski, J. Horacek, K. Krieger, A. Kukushkin, J. Likonen, G. F. Matthews, M. Rubel, and J. D. Strachan, Plasma Phys. Control. Fusion 47, B303 (2005)

${ }^{3}$ H. B. Garett, Reviews of Geophysics 19, 577 (1981)

${ }^{4}$ D. Bohm, The Characteristics of Electrical Discharges in Magnetic Fields (A. Guthrie and R. K. Wakerling, McGraw-Hill, New York, 1949) p. 77

${ }^{5}$ K. U. Riemann, J. Phys. D: Appl. Phys. 24, 493 (1991)

${ }^{6}$ D. D. Tskhakaya, B. Eliasson, P. K. Shukla, and S. Kuhn, Phys. Plasma 11, 3945 (2004)

${ }^{7}$ K.-B. Persson, Phys. Fluids 5, 1625 (1962)

${ }^{8}$ R. Chodura, Phys. Fluids 25, 1628 (1982)

${ }^{9}$ P. Stangeby, Phys. Plasmas 2, 702 (1995)

${ }^{10}$ E. Ahedo, Phys. Plasma 4, 4419 (1997)

${ }^{11}$ K. U. Riemann, Phys. Plasma 1, 552 (1994)

${ }^{12}$ S. Devaux and G. Manfredi, Phys. Plasma 13, 083504 (2006)

${ }^{13}$ D. D. Tskhakaya and L. Kos, Phys. Plasma 21, 102115 (2014)

${ }^{14}$ P. Stangeby, C. Pitcher, and J. Elder, Nuclear Fusion 32, 2079 (1992)

${ }^{15}$ P. Stangeby, Nuclear Fusion 52, 083012 (2012)

${ }^{16}$ D.Coulette and G.Manfredi, Plasma Phys. Control. Fusion 58, 025008 (2016)

${ }^{17}$ D. D. Tskhakaya, P. K. Shukla, B. Eliasson, and S. Kuhn, Phys. Plasma 12, 103503 (2005)

${ }^{18}$ N. S. Krasheninnikova, X. Tang, and V. S. Roytershteyn, Phys. Plasma 17, 057103 (2010)

${ }^{19}$ K. Theilhaber and C. K. Birdsall, Phys. Fluids B 1, 2244 (1989)

${ }^{20}$ K. Theilhaber and C. K. Birdsall, Phys. Fluids B 1, 2260 (1989)

${ }^{21}$ M. J. Gerver, S. E. Parker, and K. Theilhaber, Phys. Fluids B 2, 1069 (1990)

${ }^{22}$ D. L. Holland, B. D. Fried, and G. J. Morales, Phys. Fluids B 5, 1723 (1993)

${ }^{23}$ U. Daybelge and B. Bein, Phys. Fluids 24, 1190 (1981)

${ }^{24}$ J. Moritz, E. Faudot, S. Devaux, and S. Heuraux, Phys. Plasmas 23, 062509 (2016)

${ }^{25}$ J. Moritz, E. Faudot, S. Devaux, and S. Heuraux, Phys. Plasmas 25, 013534 (2018)

${ }^{26}$ R. Bisswell, P. Johnson, and P. Stangeby, Phys. Fluid B 1, 1133 (1989) 
${ }^{27}$ P. C. Stangeby, The Plasma Boundary of Magnetic Fusion Devices (Institute of physics Publishing, London, 2000) p. 413

${ }^{28}$ D. Tskhakaya and S. Kuhn, J. Nuc. Mater. 313, 1119 (2003)

${ }^{29}$ E. Faudot, S. Devaux, J. Moritz, S. Heuraux, P. M. Cabrera, and F. Brochard, Rev. Sci. Instrum. 86, 063502 (2015)

${ }^{30}$ J. Bak, R. Pitts, H. Kim, H. Lee, C. Bin, J. Juhn, S. Hong, O. Garcia, R. Kube, and D.C.Seo, Nuclear Materials and Energy 12, 1270 (2017) 